\title{
Fomento de la lectura a través de aplicaciones de literatura infantil en portugués: algunas propuestas
}

\author{
Belén García-Delgado Giménez ${ }^{1}$
}

Recibido: 29 de junio de 2018 / Aceptado: 31 de octubre de 2019

Resumen. Como consecuencia de la irrupción de las nuevas tecnologías y del impacto de estas en las nuevas generaciones, este trabajo propone hacer un buen uso de las mismas. En este sentido, se lleva a cabo una argumentación acerca de la importancia de la aplicación de estas en el aula, explicando así las ventajas y desventajas que presentan. Se aborda de esta manera el papel del profesor, de la escuela y de las bibliotecas. En segundo lugar, se definen los nuevos géneros de lectura que han surgido recientemente en la literatura infantil, para dar así una relación de las aplicaciones disponibles en portugués para el aprendizaje de la lectura, así como para el fomento de la lectura de obras infantiles. De esta forma, se clasifican por géneros, contenidos, edades de los destinatarios, niveles de descodificación, etc. En todos los casos, se exponen los criterios de calidad que se deben tener en cuenta a la hora de seleccionar las diferentes aplicaciones. Por último, se incluyen varias propuestas para potenciar el uso de estas nuevas herramientas en la escuela.

Palabras clave: Literatura infantil, Portugués, Aplicaciones de lectura, Enseñanza de la lectura, Fomento de la lectura.

\section{[en] Reading promotion through children's literature applications in Portuguese: some proposals}

\begin{abstract}
As a result of the proliferation of new technologies and the impact they have on new generations, this paper suggests how to make the best use of them. Accordingly, we explain the reasons why they should be used in the classroom, focusing on their advantages and disadvantages. Likewise, we also discuss the role of teachers, schools and libraries. Secondly, we define the latest reading genres that have recently emerged in children's literature in order to provide a list of the applications available in Portuguese for reading literacy purposes as well as for reading literary works aimed at children. In this way we classify the applications by genre, content, user age, reading level, etc. In all cases, we set out the quality criteria to be taken into account when selecting the different applications. Finally, we suggest different ways to promote the use of these new tools in the school.
\end{abstract}

Keywords: Children's Literature, Portuguese, Reading Applications, Reading Literacy, Reading Promotion.

\section{[fr] Promouvoir la lecture par voir d'applications de la littérature pour enfants en portugais}

Résumé. En raison de l'irrruption des nouvelles technologies et de son impact sur les futures générations, ce travail de recherche propose d'en faire bon usage. En ce sens, l'argumentation repose sur l'importance d'appliquer les nouvelles technologies en classe afin d'en expliquer les avantages et les inconvénients. Le rôle du professeur, de l'école et des bibliothèques y est donc abordé. Par la suite, il s'agit de définir les nouveaux genres de lecture apparus tout récemment dans la littérature d'enfance en vue de dresser un bilan des applications disponibles en portugais non seulement dans le domaine de l'apprentissage de la lecture mais aussi dans celui de la lecture d'ouvrages pour l'enfance. La classification se fait alors par genre, contenu, âge des destinataires, niveau de lecture, etc. Quoi qu'il en soit, les critères de qualité à prendre en compte avant de choisir les différentes applications sont clairement exprimés. En outre, des suggestions visant à promouvoir l'usage des ces outils en classe sont également formulées.

Mots-clés: Littérature pour enfants, Portugais, Applications de lecture, Enseignement de la lecture, Promouvoir la lecture.

Sumario: 1. Introducción. 2. Objetivos. 3. Productos a desarrollar. 4. Criterios de selección y técnicas de aplicación de las app en el aula. 5. Conclusiones. 6. Bibliografía.

Cómo citar: García-Delgado Giménez, Belén (2020): “Fomento de la lectura a través de aplicaciones de literatura infantil en portugués: algunas propuestas", en Didáctica. Lengua y Literatura, 32, 113-127. 


\section{INTRODUCCIÓN}

En la actualidad, el crecimiento del uso de las nuevas tecnologías es cada vez mayor, de modo que en 2019 el número de familias portuguesas que disponían de equipamientos con conexión a internet de banda ancha era de $80,9 \%$, mientras que en 2009 fue de 47,9\% (PORDATA, 2020a). Por este motivo, las fuentes de información se han multiplicado, véase el aumento tanto de libros como de otro tipo de documentos de la Biblioteca Nacional en Portugal que, en 2010 tenía 2.006.488 y en 2018 un total de 2.374.201. Sin embargo, el número de personas que consultan libros en la Biblioteca Nacional ha descendido de forma considerable en pocos años, siendo en 2018 de 31.775, mientras que en 2010 fue de un total de 46.502 (PORDATA, 2019a). Inferimos de esta manera que, cada vez son más los documentos que disponibles en formato digital, ya que además, observamos un descenso en el número de títulos inscritos en el Depósito Legal en el año 2018 con respecto al año 2008, pasando de un total de 17.331 monografías a $14.309^{2}$ (PORDATA, 2019b).

Esta evolución de los formatos de lectura ha afectado al proceso lector, lo que influye directamente en diversos aspectos del ámbito educativo. En primer lugar, cabe destacar el hecho de que en este momento el alumno ya no dispone únicamente del profesor y del libro de texto como medios de acceso al conocimiento. Es más, el porcentaje de alumnos portugueses de Enseñanza básica que utilizaron internet en 2019 fue del 54,2\%, mientras que en 2009 era de $30,10 \%$ (PORDATA, 2020b). En momentos como el actual las nuevas tecnologías han hecho posible que la enseñanza pueda continuar a distancia, sin necesidad de presencialidad alguna, asemejándose en gran medida a la educación tradicional.

Por estos motivos, en este trabajo nos vamos a centrar en el uso de las tabletas en el aula, ya que son múltiples las aplicaciones que han surgido con diferentes usos, estimándose unas 2.567.752 en enero de 2019 en Google Play, y en Appstore casi 2 millones durante el tercer trimestre del 2018 (Statista, 2019). De esta manera éstas se han ido incorporado poco a poco a los modelos educativos de los centros. Aunque, múltiples estudios afirman que los alumnos que usan estos dispositivos tienen peores resultados académicos (Payne, Greenberg y Walker, 2016), lo cierto es que hay diversidad de opiniones al respecto. Es por eso por lo que empezaremos a explicar las ventajas que ofrecen (Marqués, 2014). Primeramente, favorecen la interacción y, por tanto, el trabajo en grupo entre alumnos del mismo nivel. Son diversas las herramientas colaborativas que lo permiten: blogs, redes sociales, foros, chats, wikis, formularios electrónicos y documentos de edición colaborativa -como Google Drive-. Todas ellas posibilitan una comunicación rápida y eficaz entre iguales, fomentando además el sentido de pertenencia a una comunidad. Lo mismo ocurre desde la óptica del profesor, ya que posibilita la interacción entre colegas de las mismas características.

La portabilidad y el fácil transporte de estos artefactos son otros de los aspectos positivos a tener en cuenta. Se evitan así problemas como el almacenamiento y el depósito en los hogares, además de los problemas de salud que se derivan de los desplazamientos de los pesados libros de un lugar a otro.

Sin duda, una de las principales razones por las que las tabletas tienen tal éxito en el mercado es la multifuncionalidad. Si algo aprecian los usuarios es poder compatibilizar en un solo aparato tareas tan diferentes como: consultar el correo electrónico, escuchar la radio, leer un libro, hacer una fotografía o llamada telefónica, ver la televisión, etc. Muestra de ello es que en 2018 el 66,5\% de la población portuguesa de entre 16 y 74 años utilizó o instaló aplicaciones de internet desde su smartphone y tan solo un $1 \%$ en un libro electrónico (INE, 2019). Aprovechemos pues esta tendencia en auge, en cuanto a uso de este tipo de dispositivos, para fomentar el hábito lector en los más pequeños, en lugar de usarlo únicamente con fines de entretenimiento.

Bien es cierto que hay dispositivos que aún no han proliferado en el país luso, pero sí que va aumentando el número de lectores en formato digital. Muestra de ello son los datos del informe PISA del año 2018 en el que se afirma que los alumnos portugueses obtuvieron 492 puntos en el proceso lector en ambos formatos: papel y digital, siendo de 470 en el año 2000 (OECD, 2019). El porcentaje del nivel de destreza de alumnos de cinco a seis años que alcanza un nivel superior en lectura es de 2,5 puntos más que en el informe anterior del año 2016.

Otra de las competencias que se fomentan con el uso de estos dispositivos desde el punto de vista educativo es el aprendizaje autónomo, ya que son múltiples las herramientas disponibles para la realización de actividades de diferentes materias. Gracias a las mismas, el alumno tiene la posibilidad de evaluar sus conocimientos sin ayuda de mediadores, por lo que puede ser un elemento de apoyo para el alumno que tenga dificultades en ciertas asignaturas. Cada vez proliferan más aplicaciones como Smartick, en este caso para el aprendizaje de matemáticas, llegando a ocupar el primer lugar en número de descargas en aplicaciones educativas de origen español ${ }^{3}$.

Muy importante también es la creatividad, destreza que se puede incentivar a través de la comunicación oral, la expresión escrita, la pintura, la fotografía, etc. En este sentido, el hecho de que la difusión de cualquier producto, creado o elaborado por el alumno, se multiplique gracias a la web, puede resultar alentador.

Por estos motivos, cada vez son más las escuelas portuguesas de enseñanza básica que disponen de ordenadores con conexión a internet, 163.841, lo que supone 92,2\% del total (PORDATA, 2019c). Desconocemos si en estas

Dado que el Decreto Ley de Depósito Legal de Portugal en vigor data de 1982 (Governo de Portugal, 1982), las publicaciones en formato digital no se contemplan en esta regulación.

3 Una de las razones de su éxito es que, además de motivar el aprendizaje de forma independiente sin necesidad de ayuda alguna por parte de padres o tutores, incluye actividades breves de 15 minutos, tiempo medio de concentración de un niño, como ellos mismos afirman en su portal web. Más información en: https://www.smartick.es/. 
cifras se tienen en cuenta también otro tipo de dispositivos con acceso a internet como son las tabletas, ya que no se distinguen cifras en este sentido.

Pero, por los datos mencionados anteriormente, parece que a pesar de que disponen de la infraestructura adecuada y de que el uso de la web está aumentando, este aún no es mayoritario. Esto puede deberse a que aún son pocos los materiales en lengua portuguesa publicados en la web, lo que puede ser una gran dificultad para los alumnos de corta edad que aún no disponen de un nivel suficiente en lenguas extranjeras como el inglés. Si a esto añadimos que, además de ser un país de reducida extensión y población $(92,391 \mathrm{~km}$. y 10.196.709 habitantes según datos de Internet World Stats, 2020) solamente un $13,9 \%$ de sus residentes tiene menos de 15 años - siendo de 15,6\% la media de la Unión Europea (PORDATA, 2019e)- podremos entender los motivos por los que no se invierte en crear herramientas para los menores en lengua portuguesa. También es reseñable que el porcentaje de gasto del PIB que el país luso invierte en investigación es de $0,7 \%$, más de un punto por debajo de la media de la Unión Europea (PORDATA, 2020b).

Lo cierto es que, a pesar de que es frecuente observar a niños que manejan tabletas mejor que los adultos, cuando se trata de leer el 69\% de los niños de 6 a 8 años prefiere leer libros en papel, incluso si hay libros electrónicos disponibles (Scholastic, 2016). No disponemos de estudios que analicen el uso de los diferentes dispositivos por parte de los menores en Portugal pero, en España un 26,2\% de los niños de 6 a 7 años y un 28,3\% de los de 8 a 9 años prefieren usar un Tablet o iPad frente a un 15,8\% y un 14,3\% -respectivamente- que elige el móvil como dispositivo (AIMC, 2018). Esto nos lleva a pensar que "tal vez no estamos aprovechando bien esa fascinación por las pantallas para convertir las aplicaciones de lectura en una herramienta para fomentar el hábito lector" (García-Rodríguez; Gómez-Díaz, 2016). En este sentido la labor de los mediadores es fundamental.

Son muchos los autores que defienden las bondades de las tabletas en cuanto a la eficacia y eficiencia en el rendimiento académico de los estudiantes. Los motivos son, entre otros, la comodidad y facilidad de uso, ya que en su mayoría están habituados a utilizarlos a diario. Es más, como valor añadido cabe destacar la difusión social de las competencias digitales, ya que estas pueden transmitirse a los padres, suscitando así interés por el manejo a los mismos.

Lo cierto es que pedagógicamente el potencial de estos aparatos es enorme, muchas son las nuevas metodologías didácticas, centradas en el alumno, que se pueden aplicar. Véase por ejemplo flipped classroom, aprendizaje por competencias, realidad aumentada, mobile learning, diseño de aplicaciones, etc.

Pero, no todo son ventajas, también existen inconvenientes, tal y como exponemos a continuación (Marqués, 2014). En primer lugar, la tecnología no siempre funciona adecuadamente, por lo que una mala conexión a internet, o la incompatibilidad de diferentes dispositivos puede provocar que una clase no se pueda desarrollar tal y como estaba prevista.

Por otro lado, el hecho de que haya que abrirse una cuenta para la descarga de algunas de las aplicaciones, dificulta el manejo de las tabletas por parte de los más pequeños. Esto se debe a que para registrarse se requieren datos de los que los niños no disponen, por lo que de nuevo interrumpe el ritmo de la clase.

$\mathrm{Si}$ a esto añadimos el hecho de que las aplicaciones de contenido educativo se encuentran en muchas ocasiones únicamente en lengua inglesa, el público objetivo puede llegar a reducirse enormemente.

Otras veces ocurre que los docentes no tienen tiempo para desarrollar los nuevos recursos tecnológicos o para formarse en el manejo de los mismos.

Se debe tener en cuenta también que, debido a la versatilidad de estos dispositivos, son muchas las distracciones que pueden llegar a darse, ya que las aplicaciones y usos son variados. Por lo que, esa versatilidad o multifuncionalidad que enunciábamos anteriormente como ventaja a la hora de seleccionar un dispositivo, puede llegar a ser una desventaja también si no se usan correctamente.

La tecnología tiene sus limitaciones, ya que, si por ejemplo un día un alumno se olvida de traer a clase la tableta, se le acaba la batería porque no lo ha cargado en casa, o no ha actualizado las aplicaciones, el desarrollo de la clase puede llegar a alterarse.

Por último, también debemos señalar que la obsolescencia tecnológica se traduce en que, al cambiar de forma frecuente los formatos y dispositivos, el coste económico para las familias y centros sea elevado y además, los mediadores precisen de una actualización constante en alfabetización digital.

Aparte del profesor, otro de los principales mediadores para el uso educativo de las tabletas es el bibliotecario. El problema es que el número de bibliotecas escolares en Portugal, aunque va aumentando año tras año, es aún escaso -en 2017 la red de bibliotecas escolares del sector público y privado se componía de un total de 2.461 (PORDATA, 2019f)-. Sin duda, la biblioteca escolar tiene gran importancia en este sentido, ya que según el Arts Council (2013) estos centros deben aprovechar las nuevas tecnologías digitales poniendo las mismas a disposición de los usuarios: libros autopublicados por los propios autores, aplicaciones para dispositivos móviles y contenidos generales para usuarios como: videojuegos, música y cine.

En este sentido Lectura Lab da algunas recomendaciones para las bibliotecas escolares infantiles como son las siguientes (2010). En primer lugar, aumentar la oferta de contenidos para niños y jóvenes con libros electrónicos, música y aplicaciones de todo tipo. En segundo lugar, realizar actividades de alfabetización digital como talleres y cursos para el uso de los dispositivos. También destaca la importancia de tener un servicio de préstamo de contenidos digitales de todo tipo y ofrecer actividades donde usar diferentes soportes para poder leer libros digitales.

Las plataformas de contenidos referenciales o redes sociales de lectura son de gran utilidad para el mediador, ya que realizan recomendaciones, clasificaciones de las obras por edad, formato, competencias, temática, etc. De esta 
forma se dispone de una gran ayuda a la hora de seleccionar una obra determinada para unos destinatarios concretos. Así, encontramos recomendaciones, guías de lectura, reseñas de libros en: Common Sense Media, Babelio, Canada's Centre por Digital and Media Literacy, Goodplayguide y Leoteca. En lengua española e inglesa disponemos de diversidad de herramientas, pero en portugués, o que incluyan referencias a libros en lengua portuguesa, encontramos pocas. Goodplayguide describe, analiza y recomienda, en inglés, aplicaciones de lectura infantil en muchas lenguas, entre ellas el portugués tanto de Brasil como de Portugal. Leoteca también hace reseñas en portugués de libros electrónicos en esta misma lengua, para niños y jóvenes. Pero, no dispone de información alguna de aplicaciones de lectura en portugués.

En lengua portuguesa de Brasil encontramos la Plataforma do letramento, que incluye diversas actividades de comprensión lectora, ideas para el fomento de la lectura en la escuela, reseñas de obras e incluso, aportaciones de mediadores para la aplicación de actividades en una obra concreta en la escuela.

Por último, debemos mencionar el proyecto ler+ de Portugal enmarcado dentro del Plano Nacional de Leitura, que tiene la misma función que la Plataforma do letramento pero en este caso en Portugal. Lo curioso de esta plataforma es que describe numerosos recursos para la lectura, como por ejemplo bibliotecas virtuales, blogs sobre lectura, repositorios y bases de datos, pero en su gran mayoría estas están disponibles en español -Leer en pantalla, Dosdoce literatura digital, etc.- o en inglés -Easypeasy app, Shanahan on Literacy, etc.-, pero no en portugués.

Lo cierto es que cada vez se publica menos en portugués, ya que en el formato tradicional el número de títulos descendió de 17.379 en 2008 a 8.072 en 2018 y, el número de títulos en portugués traducidos de otras lenguas en 2018 fue de 2.782, siendo de 3.798 en 2008 (PORDATA, 2019d).

\section{Objetivos y metodología}

Como hemos visto, autoridades diversas, tanto expertos como organizaciones, defienden y aconsejan el uso de las nuevas tecnologías en la escuela y en las bibliotecas infantiles y juveniles. Lo que debemos lograr ahora es que además de ser útiles en la enseñanza, "pasen de ser competidores a aliados de la lectura" (García-Rodríguez, GómezDíaz, 2017).

Por eso, el objetivo de este trabajo es exponer una relación de algunas de las herramientas tecnológicas educativas, poniendo el foco en la tipología de aplicaciones disponibles para la enseñanza de la lectura infantil en lengua portuguesa, así como aquellas que fomenten las competencias de expresión oral y expresión escrita. Estos documentos pueden emplearse para fomentar la lectura tanto de niños y jóvenes, como de adultos que estudien portugués como lengua extranjera.

Se trata de un idioma poco popular en la red de redes en comparación con otros como el inglés o el español. Sin embargo, esta característica es especialmente importante en el caso del público infantil que, al encontrarse en pleno desarrollo de la competencia oral y escrita de su lengua materna, debe poder acceder a contenidos en la misma. De ahí el interés por darlas a conocer y clasificarlas adecuadamente según el tipo de aplicaciones. En este caso, después de explicar la diferente tipología que existe, desarrollaremos solamente aquellas en las que encontramos ejemplos de títulos en lengua portuguesa. La metodología de recuperación de las mismas ha sido realizar búsquedas en aquellas plataformas de popularidad y prestigio, donde tanto usuarios como expertos recomiendan las diferentes obras, como son: Goodplayguide, Chiquimedia y Leoteca. En la mayoría de los casos estas herramientas no suelen disponer de una búsqueda avanzada por lengua, por lo que el proceso no ha sido sencillo. Además se ha intentado comprobar si aquellos productos recomendados en español en los distintos artículos de investigación, también están disponibles en portugués.

De esta forma tendremos: libros app, las aplicaciones de storytelling, las de reconocimiento de sonidos con grafías y aquellas destinadas a niños con capacidades especiales.

En cada una de las muestras aportadas se explicarán algunos de los factores de selección que pueden ser importantes para el mediador como: la edad de los destinatarios, los contenidos, las actividades que incluyen y la forma de adquisición (si exige o no suscripción, si incluye algún libro de muestra o, se puede leer de forma gratuita con publicidad). Para facilitar la identificación de cada una de ellas se incluye el icono, así como la URL para descargarla en los diferentes sistemas operativos, lo cual facilita el uso en el aula sin excluir ningún tipo de marca o modelo (García Rodríguez y Gómez Díaz, 2015).

\section{Productos Desarrollados}

Como hemos explicado en líneas anteriores, son muchas las posibilidades que ofrecen las nuevas tecnologías en la enseñanza. Por este motivo, a continuación enumeramos algunos de los productos que se pueden utilizar en las escuelas para el desarrollo de las competencias oral y escrita en lengua portuguesa, con el fin de fomentar así el gusto por la lectura.

Los blogs de la biblioteca escolar - con anuncios de las actividades del centro, recomendaciones de libros, etc.pueden ser una herramienta muy útil de comunicación entre los alumnos y el propio centro. 
Los beacons ${ }^{4}$ son también muy prácticos ya sea para el envío instantáneo de notificaciones al usuario sobre eventos o recomendaciones o para el servicio de préstamo.

Los audiolibros digitales, aunque fueron creados para personas con capacidades especiales, su uso se ha extendido de forma mayoritaria debido a sus posibilidades pedagógicas. Se trata de grabaciones narradas o leídas automáticamente por programas informáticos que pueden ser especialmente útiles para niños que están aprendiendo a leer.

Un producto más actual sería el video libro o video narración, el cual se puede presentar en formatos diferentes. Ejemplo de ello serían los libros app en los que diferentes historias se reproducen en vídeos personales (García Rodríguez y Gómez Díaz, 2016).

De esta variedad encontramos varias modalidades en lengua portuguesa como son:

- Auca: libro app, creado por pedagogos, para niños de 6 a 8 años, con 3 niveles de lectura disponibles. Los cuentos no están únicamente en portugués (de Brasil), sino en más lenguas. El tipo de letra se puede modificar, adecuándose así a las necesidades de los destinatarios. Posee además animaciones, ilustraciones y banda sonora, disponiendo de actividades de comprensión lectora al final ${ }^{5}$. La aplicación es gratuita, pero la descarga de libros no lo es. Eso sí, se ofrece un libro gratuito de muestra en español.

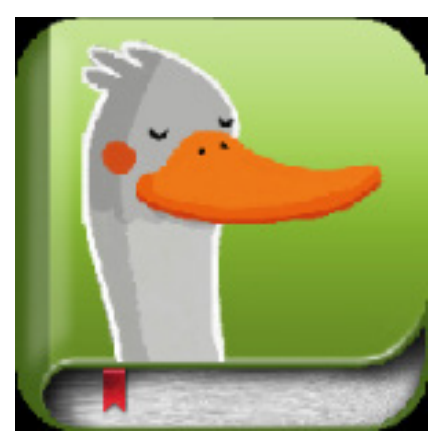

Figura 1. Logo de Auca (Aucadigital, 2017)

- Children's Tales: aplicación de lectura para niños de 0 a 12 años. Tiene series de películas para bebés que se pueden ver offline. Ofrece la posibilidad de hablar de la historia después de la lectura ${ }^{6}$. El acceso es libre, al igual que algunas de las películas y libros.

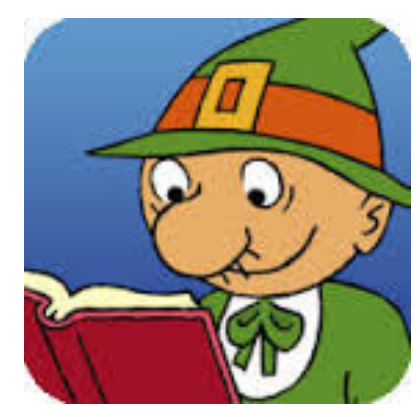

Figura 2. Logo de Children's Tales (BCNmultimedia, 2017)

- Buenas noches HD - La aplicación para niños a la hora de dormir: libro app para niños de hasta 5 años. Tiene ilustraciones 3D con animaciones y 13 animales interactivos ${ }^{7}$. Es de acceso abierto para Android, pero no para Apple. Los libros que incluye sí son de pago, tan solo es gratuita una pequeña muestra de un libro. Resulta ideal para todos aquellos padres que deseen crear una rutina de sueño en sus hijos.

\footnotetext{
Según Forrest Stroud, "beacons are small, often inexpensive devices that enable more accurate location within a narrow range than GPS, cell tower triangulation and Wi-Fi proximity. Beacons transmit small amounts of data via Bluetooth Low Energy (BLE) up to 50 meters, and as a result are often used for indoor location technology, although beacons can be used outside as well" (http://www.webopedia.com/TERM/B/beacon.html, consulta: 29 de junio de 2017).

Más información en: http://aucadigital.com/index.php/es/auca_es/.

6 Disponible para iPhone y Smartphone:

https://itunes.apple.com/us/app/childrens-tales-educational/id375755924?mt=8,

https://play.google.com/store/apps/details?id=com.greatappwarehouse.cuentosinfantiles\&hl=en.

7 Disponible para iPhone y Smartphone:

https://itunes.apple.com/es/app/buenas-noches!-hd-la-aplicacion/id428492588? mt=8,

https://play.google.com/store/apps/details?id=com.foxandsheep.nightynight\&hl=es_419.
} 


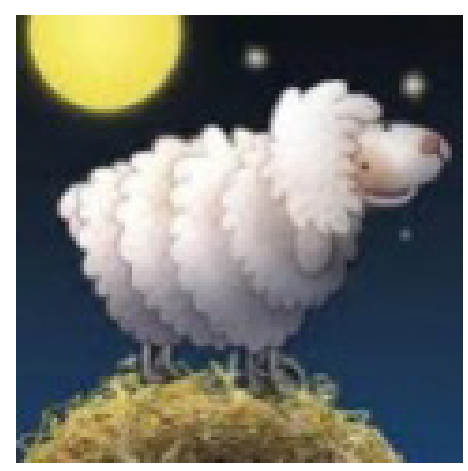

Figura 3. Logo de Buenas noches HD (Fox y Sheep, 2017).

- Nurot: libro app para destinatarios de entre 3 y 9 años. Ofrece la posibilidad de interactuar con personajes, escuchar música, mover objetos, resolver puzzles y animaciones ${ }^{8}$. Fomenta la comunicación a través de temas diversos como por ejemplo el amor a primera vista. Tiene además juegos de memoria, puzzles, etc. Para desarrollar la comprensión lectora. La descarga del libro app es libre, al igual que el acceso a parte del contenido, pero no al texto completo.

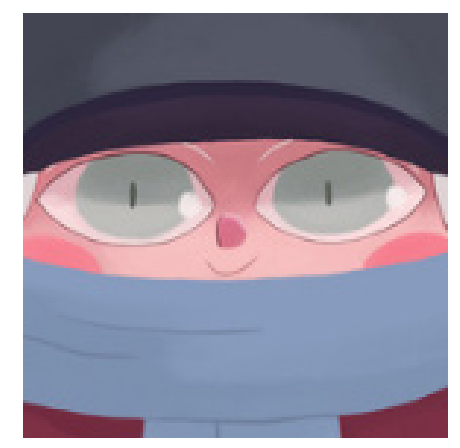

Figura 4. Logo de Nurot (Chiquimedia, 2017)

- Lucy \& Pogo-Interactive Storybook: libro interactivo recomendado para niños de 3 a 5 años. Motiva el desarrollo de la capacidad de escucha y atención y además, enseña valores como la empatía, prejuicios y tolerancia introduciendo el humor como elemento diferenciador. Tiene además, actividades de comprensión lectora. Exige un pago único de acceso.

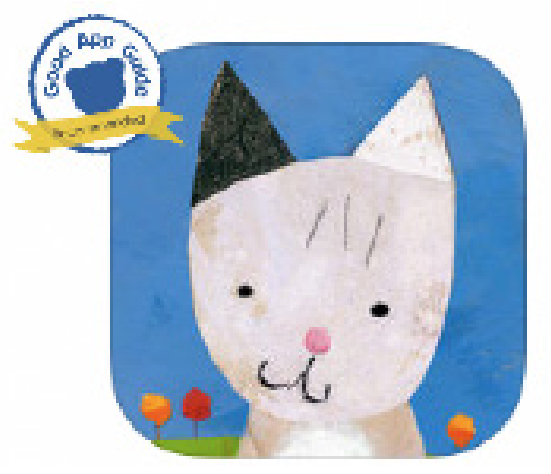

Figura 5. Logo de Lucy \& Pogo-Interactive Storybook (Goodplayguide, 2020a)

- Clean and Bright: libro app para niños de 3 a 9 años. Se compone de una historia sobre las dificultades que encuentran los niños a medida que crecen. Se puede interactuar con varios elementos de la historia, animaciones activas y sonidos. También tiene actividades de comprensión lectora como: juegos de memoria, puzzles, etc. ${ }^{9}$ Es de la misma editora que Nurot, por lo que la adquisición del software y del contenido es similar. 


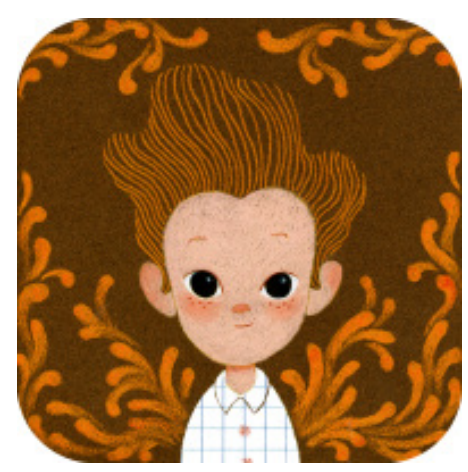

Figura 6. Logo de Clean and Bright (Chiquimedia, 2017)

- The Platypus'Search: aplicación interactiva destinada a niños de entre 3 y 4 años que están aprendiendo a leer, ya que motiva la enseñanza de vocabulario a través de la escucha y construcción de palabras. Fomenta la concentración del niño a la hora de interactuar en la historia, además de valores como la diversidad y los prejuicios. Disponible para dispositivos de Apple, previo pago de una única tarifa.

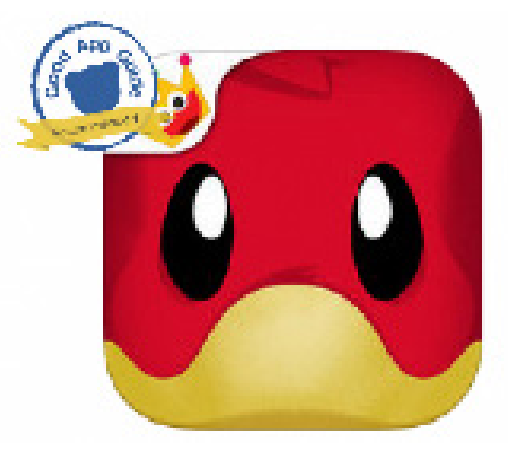

Figura 7. Logo de The Platypus'Search (Googdplayguide, 2020b).

- Nighty Night Circus: aplicación que ayuda a aprender y construir vocabulario, así como a desarrollar habilidades de comunicación oral. Está destinada, por tanto, para niños de hasta 5 años. Se precisa del pago de una única cuota para acceder. Fomenta la adquisición de rutinas diarias a la hora de irse a dormir, así como la observación e interacción con los personajes de la historia ${ }^{10}$.

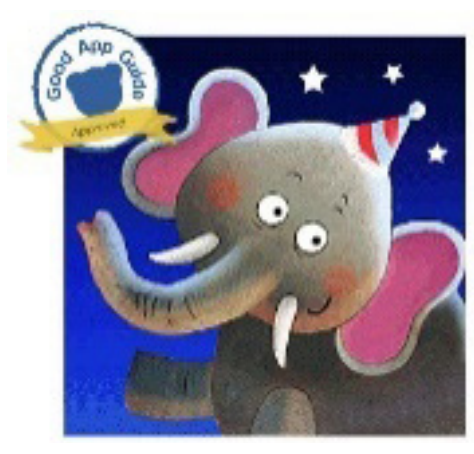

Figura 8. Logo de Nighty Night Circus (Goodplayguide, 2020c).

Otra variedad de esta misma tipología serían los libros presentes en plataformas de vídeo como Youtube o Vimeo, donde se emiten reproducciones animadas de cuentos clásicos. También encontramos títulos originales en la web.

La realidad aumentada, presente en literatura infantil y juvenil, está teniendo gran éxito entre los niños y jóvenes. Se trata de tecnología de superposición en tiempo real, que también puede presentarse en diferentes formatos. Uno de ellos son los libros en papel con marcadores para el uso de la aplicación, donde el libro físico incorpora marcadores (códigos QR) que dan acceso a material complementario a la historia. Este material puede ser vídeo, imagen, o incluso alguna pista para desentrañar el argumento del propio libro. Otra variedad sería la lectura del libro a través del ordenador per- 
sonal donde, con un programa informático, los contenidos extra se visualizan a través de una webcam. En último lugar encontramos los libros con marcadores con contenidos en 3D que se activan con un gadget de un videojuego.

Por otro lado, las app toy o apptivit toy, es decir, los complementos físicos o juguetes para interactuar con una determinada aplicación, son cada vez más frecuentes. Es una estrategia de venta, ya que presentan un valor añadido al propio libro.

Deducimos así que la ergonomía, en cuanto a legibilidad, claridad e interfaz se refiere, es una de las características mejor valoradas a la hora de seleccionar un objeto de lectura.

Por eso, las aplicaciones con fines literarios son cada vez más numerosas, llegando a abarcar todo tipo de géneros, incluso los cómics, de gran éxito entre los jóvenes. Siempre se debe tener en cuenta que, si bien estas aplicaciones aproximan tanto a niños como a jóvenes a la lectura, lo más relevante a la hora de seleccionar un documento u otro es el contenido y no, lo que acompaña al mismo. En cualquier caso, este tema sería objeto de estudio de otro artículo.

Otra posibilidad son las aplicaciones para crear tus propios contenidos, las cuales pueden ser de gran ayuda, para mediadores en escuelas y bibliotecas, a la hora de producir los contenidos que se deseen. Aurasma ${ }^{11}$ y Layar $^{12}$ son claros ejemplos de ello.

En la misma línea encontramos las aplicaciones de storytelling, que permiten crear historias y cuentos digitales, además de grabar vídeos, incorporar música, utilizar nuestra voz o incluir un texto o ilustraciones propias (García Rodríguez y Gómez Díaz, 2016). Se trata pues de otra forma de dar protagonismo al lector, permitiendo que interaccione a través del movimiento de objetos, personajes, sonido, banda sonora, etc., haciéndolo así parte de la historia. También es interesante que se ofrezca la posibilidad de personalizar los contenidos, pudiendo así customizarlos según las preferencias del usuario.

De esta tipología en lengua portuguesa tenemos:

- Marina y la luz: cuento interactivo, para niños de hasta 5 años, con fuerza visual y banda sonora. Los niños pueden crear una historia diferente cada vez. Valores como la tolerancia, la libertad, la igualdad, el compromiso y la fraternidad son transmitidos en la historia. Es útil para estimular la creatividad, la alfabetización visual, compartir experiencias, etc. ${ }^{13} \mathrm{El}$ acceso no es gratuito.

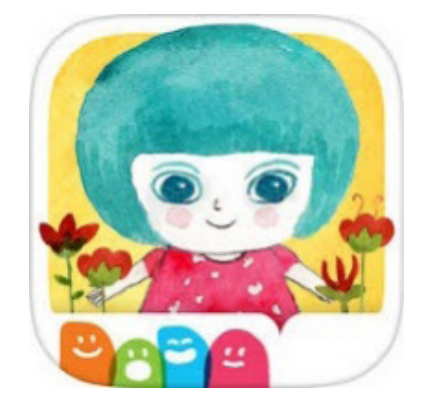

Figura 9. Logo de Marina y la luz (Dadacompany, 2017)

- Dr. Panda Ciudad: Vacaciones: Versión completa de pago sin límite de uso y además, dispone una versión parcial gratuita. Es recomendable para niños de entre 4 y 6 años. Motiva la curiosidad de los niños, así como la capacidad para contar historias. Eso sí, se encuentra en portugués de Brasi1 ${ }^{14}$. De la misma serie podemos encontrar: Dr Panda Restaurant.

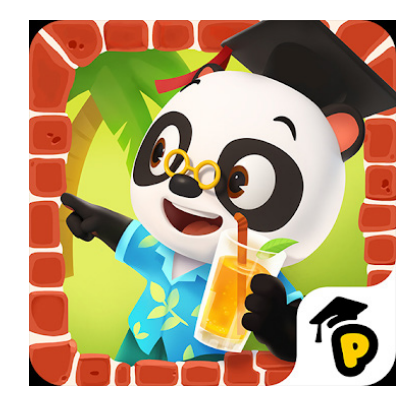

Figura 10. Logo de Dr. Panda Ciudad: Vacaciones (Goodplayguide, 2020d)

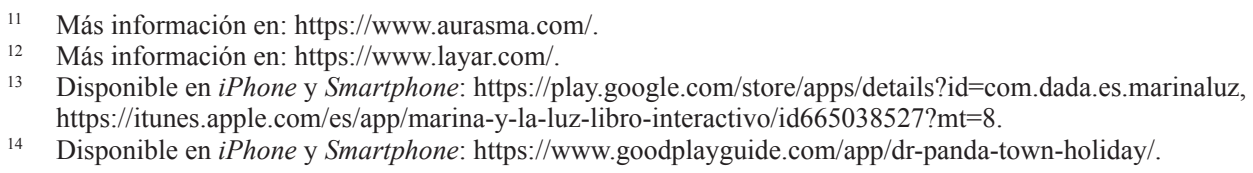


- Me by Tinybop: aplicación de pago para niños de entre 6 a 8 años, en la que se les motiva a contar historias acerca de sus gustos, así como acerca de su identidad y relaciones ${ }^{15}$. Se desarrollan así múltiples habilidades como la creatividad, toma de decisiones, trabajo en grupo, construcción de vocabulario, comunicación oral y manejo de sentimientos y emociones.

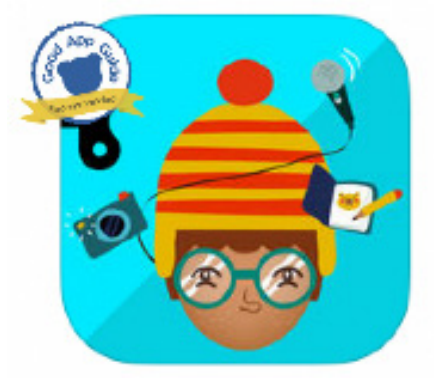

Figura 11. Logo de Me by Tinybop (Goodplayguide, 2020e)

- Tiggly Stamp: esta aplicación de acceso gratuito está indicada para niños de hasta 4 años . Ofrece la posibilidad de crear una película al arrastrar elementos a la historia y participar así en la creación del nuevo argumento de la misma. Fomenta la adquisición de palabras nuevas de animales, frutas y objetos.

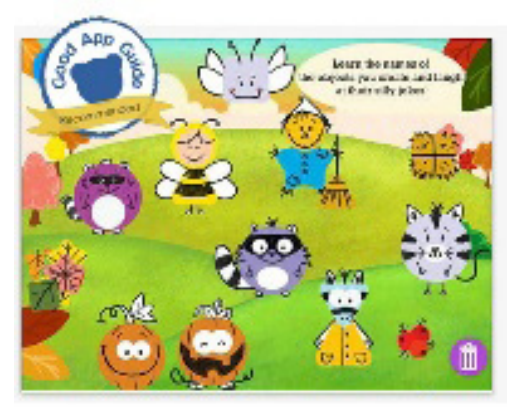

Figura 12. Logo de Tiggly Stamp (Goodplayguide, 2020f)

- Princess Drawsalot And The Dragon: aplicación para niños de entre 3 a 10 años que requiere un pago único de acceso. En ella se motiva a los usuarios a usar su imaginación al pintar y contar sus propias historias, desarrollando así la creatividad, el reconocimiento de formas, colores, espacios y medidas ${ }^{16}$. Además, ofrece luego la posibilidad de poder ver sus propias producciones hechas realidad.

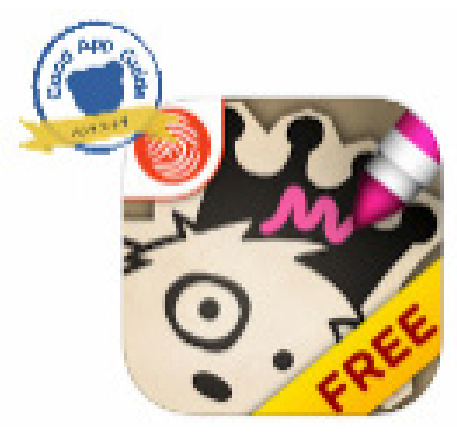

Figura 13. Logo de Princess Drawsalot And The Dragon (Goodplayguide, 2020g)

- Special Stories: el acceso a esta aplicación en versión completa requiere pagar una cuota única. Está destinada a niños de entre 5 y 7 años. Fomenta el vocabulario y la comunicación oral a la hora de organizar el discurso para hablar de rutinas y planear acciones futuras ${ }^{17}$.

15 Disponible en iPhone. https://www.goodplayguide.com/app/me-by-tinybop/.

16 Disponible para dispositivos Apple, Android y Kurio. Más información en: https://www.goodplayguide.com/app/princess-drawsalot-and-the-dragon/.

17 Disponible en iPhone y Smartphone: https:/www.goodplayguide.com/app/special-stories/. 


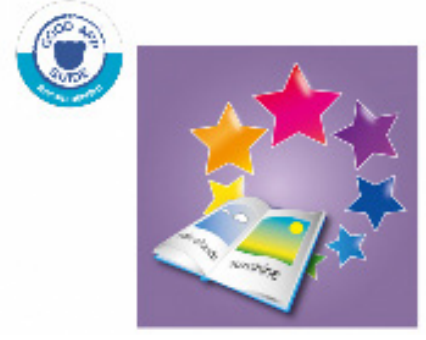

Figura 14. Logo de Special Stories (Goodplayguide, 2020h)

- Toca Life: Hospital: esta aplicación, que requiere de un pago único de acceso, está destinada a niños de entre 4 y 6 años. Ayuda a los niños a entender el ciclo de la vida a través de la interacción entre los distintos objetos de las diferentes áreas de un hospital. Al ser de final abierto, permite que los lectores inventen sus propias historias, potenciando así su creatividad, sin la presión de tener que ganar o perder un juego. Está indicado para los niños que tengan que enfrentarse a alguna enfermedad de alguien de su entorno más cercano ${ }^{18}$.

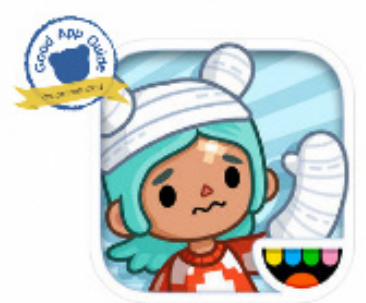

Figura 15. Logo de Toca Life: Hospital (Goodplayguide, 2020i).

- Easy stop-motion studio: esta app, para niños de más de 4 años, permite que el usuario cree su propia película a partir de fotos propias, dando la posibilidad de seleccionar la banda sonora y los subtítulos que desee. Además, puede compartirlas a través de redes sociales como Facebook, Youtube o correo electrónico ${ }^{19}$. No es de acceso libre.

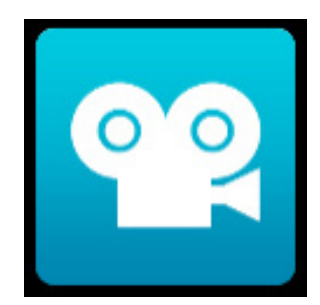

Figura 16. Logo de Easy stop-motion studio (Edokiacademy, 2017)

- Easy Studio - Introduction to animation Halloween special: del mismo estilo que la anterior, esta aplicación permite a los lectores de 6 a 8 años crear su propia película animada. Gracias a un sencillo tutorial los niños pueden crear y narrar su propia historia haciendo uso de su propia imaginación y dotes creativos. El acceso no es libre.

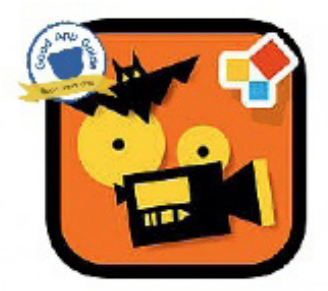

Figura 17. Logo de Easy Studio (Goodplayguide, 2020j)

18 Disponible para dispositivos de Apple, Android y Amazon, más información en: https://www.goodplayguide.com/app/toca-life-hospital/. Disponible en iPhone y Smartphone:

https://itunes.apple.com/es/app/easy-stop-motion-studio/id593847174?mt=8,

https://play.google.com/store/apps/details?id=com.cateater.stopmotionstudio\&hl=es_419 
- Tiny Builders - Digger, Crane and Dumper for Kids! Aplicación en la que los niños de 2 a 5 años tienen la posibilidad de narrar lo que ocurre en la propia historia a través de la exploración e interacción con los distintos elementos de la construcción. Incluso pueden customizar a los personajes a su antojo. Para acceder a la misma se requiere de un único pago. Está disponible únicamente para dispositivos Apple ${ }^{20}$ en portugués de Brasil. Dentro de la misma colección de aplicaciones protagonizadas por Tiny encontramos otras como: Tiny Airport, útil para el aprendizaje de vocabulario relacionado con el aeropuerto

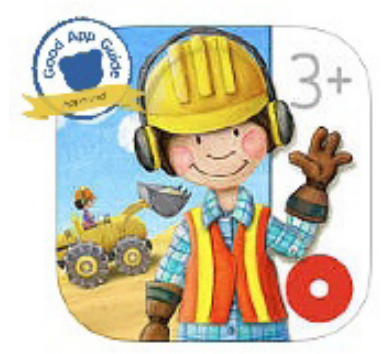

Figura 18. Logo de Tiny Builders (Goodplayguide, 2020k)

- Mortimer and the Dinosaurs: esta aplicación está recomendada para niños de entre 3 a 9 años. Se caracteriza por carecer de texto, tan solo imágenes, motivando así a los padres y a los niños a narrar activamente la historia. Gran variedad de elementos interactivos contribuyen en este sentido, junto con una banda sonora original y actividades de comprensión al final (puzzles, juegos de diverso tipo como: de memoria, de emociones y de adivinar la escena siguiente de la historia) ${ }^{21}$. El sistema de adquisición de esta app es similar al de Nurot y al de Clean and Bright, ya que son de la misma editora.

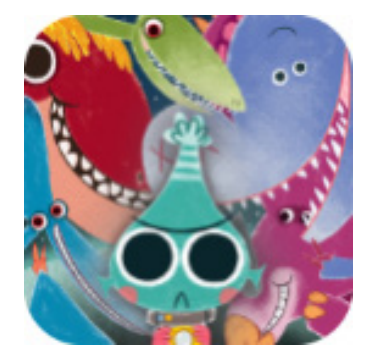

Figura 19. Logo de Mortimer and the Dinosaurs (Chiquimedia, 2017)

Si se quieren utilizar estas aplicaciones de storytelling en el aula o biblioteca se puede activar el movimiento de objetos, sonido, personajes y banda sonora; realizar una grabación personalizada de la historia, o jugar con los personajes de los libros a través de aplicaciones basadas en ellos.

Otra posibilidad en este sentido sería usar la técnica de narración donde el bibliotecario o profesor puede contar la historia en voz alta mientras que los niños ven las imágenes en la pantalla. Para ello, hay herramientas como: Storybook Reading ${ }^{22} \mathrm{u}$ otras sin locución y texto. También puede ser que la tableta cuente la historia y que sea el narrador el que incorpore la voz en la app al mismo tiempo que se reproduce en la pantalla, o usa la voz sin texto.

Son interesantes además las aplicaciones para aprender el alfabeto, tanto para nativos como para extranjeros, en las cuales se practica la fonética de una lengua, sus grafías, su ortografía, etc. Para ello, se presentan actividades diversas con el fin de completar palabras, diseñar y pintar letras, etc. También hay otras para relacionar las letras con diferentes objetos o animales a través de juegos de reconocimiento y asociación, incluyendo además la fonética de cada palabra y ejercicios de audio para reforzar (García Rodríguez y Gómez Díaz, 2016). Un ejemplo de estas últimas en portugués sería:

- Teach Your Monster to Read: Aplicación animada indicada para los niños de 3 a 6 años que están aprendiendo a leer. Especialmente indicado para aprender a identificar las diferentes grafías con el correspondiente sonido de cada una de ellas. Los niños deben crear su propio monstruo y llevarlo a lugares mágicos e insospechados donde, a medida que aprende a leer, irá obteniendo recompensas. Está disponible en los dispositivos de Apple ${ }^{23}$.

\footnotetext{
Para más información: https://www.goodplayguide.com/app/tiny-builders/.

Disponible para iPhone y próximamente para Smartphones: https://itunes.apple.com/app/id1166865060?1=en.

Herramienta donde el mediador puede grabarse narrando la historia y acompañarlo con fotos leyéndolo, así como pantallazos del objeto de lectura. Las páginas se pasan automáticamente, por lo que el niño no precisa de realizar nada (Yuste, 2014).

23 Más información en: https://www.goodplayguide.com/app/teach-your-monster-to-read-first-steps/.
} 


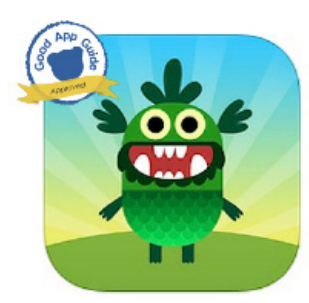

Figura 20. Logo de Teach Your Monster to Read (Goodplayguide, 20201)

Además, encontramos aplicaciones para niños que están aprendiendo a escribir y a leer. Por un lado, tenemos unas para aquellos que ya conocen el alfabeto y comienzan a deletrear y a formar sílabas y palabras, con actividades adecuadas para este proceso. Por otro, para los alumnos que comienzan a escribir hay aplicaciones con actividades de reconocimiento de las grafías de las letras, sílabas y palabras. Estas se combinan con ejercicios de escritura en los que hay juegos con fonemas, audio y la posibilidad de formar diferentes palabras con sus letras. Una muestra de ello son los diferentes cuentos de Auca dentro de la serie: Enséñame a leer.

Cuando ya saben escribir, el siguiente paso sería practicar la ortografía, para ello hay app específicas donde pueden aprender a hacerlo correctamente.

En cuanto a la comprensión lectora, encontramos apps de lectura infantil y libros app con historias adaptadas a todos los gustos e intereses infantiles, en las que se trabaja la comprensión lectora, se refuerza el proceso lectoescritor y se enriquece y amplía el vocabulario.

Para la práctica de la lectoescritura hay juegos con palabras como: crucigramas, ahorcado, apalabrados, etc.

Como no podía ser de otra manera, para los alumnos con capacidades especiales hay aplicaciones inclusivas que permiten adaptar las necesidades de los alumnos conforme a sus características y circunstancias. Se adaptan así al ritmo de aprendizaje de cada alumno, incidiendo en aspectos en los cuales tienen dificultades. No podemos olvidar que en todos los casos la organización de los contenidos es muy importante, adecuándose así la interfaz a las necesidades de la persona que las usa pero aquí, en el caso de niños con capacidades especiales, es aún más relevante.

- Tembo el elefante: aplicación de la editorial BubbleBooks para niños - con autismo, dislexia, TDAH, dificultades visuales...- a partir de 3 años. El objetivo de la misma es reforzar la atención y la memoria a través de una cuidada redacción y vocabulario del texto. La interacción con los elementos de la historia fomenta la identificación con los animales del cuento ${ }^{24}$. Únicamente disponible para Android, no para Apple, previo pago de una tasa.

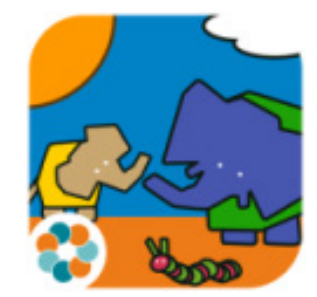

Figura 21. Logo de Tembo el elefante (BubbleBooks, 2017)

- Special Words: Recurso para niños, de entre 4 y 6 años, que se distraen fácilmente. se adapta a las necesidades de cada niño, instigándolos a practicar la comunicación oral, así como la capacidad de asociación -a través de la asociación

- entre palabras, imágenes y sonidos-. Posee una versión a texto completo que es de pago ${ }^{25}$. Para este tipo de destinatarios puede ser muy útil, como actividad para después de la lectura, imprimir la figura de uno o más de los personajes del cuento - en el caso de que se disponga de impresora 3D- para que de esta forma lo virtual pase a ser real.

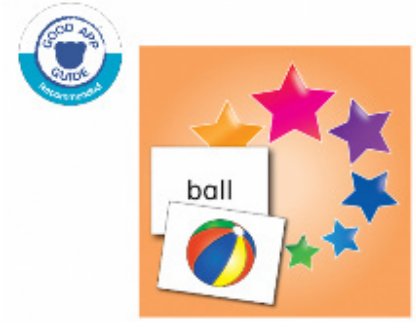

Figura 22. Logo de Special Words (Goodreads, 2020m) 


\section{CONCLUSIONES}

Son múltiples pues las posibilidades pedagógicas que ofrecen estas herramientas.

Los mediadores deben saber seleccionarlas, recomendarlas, difundirlas y aplicarlas de forma adecuada en el aula. Si se utilizan correctamente, fomentan el desarrollo de competencias y habilidades relevantes para el desarrollo del niño. De ahí la importancia de la existencia de herramientas de recomendación, valoración y clasificación de las mismas, con el fin de poder servir de apoyo a los mediadores. En español disponemos de múltiples plataformas y redes de lectura social, herramientas de préstamo entre lectores, sistemas de escritura social, clubes de lectura, comunidades de socialización, etc. Todas ellas favorecen la divulgación y posterior acceso a las distintas aplicaciones desde los hogares y las escuelas de España e Hispanoamérica. Es más, son plataformas con las que, sobre todo, los jóvenes se sienten cómodos, ya que tienen la posibilidad de interaccionar comentando (García-Rodríguez, 2015), recomendando o votando una obra que les ha gustado, y además, la difusión es mucho mayor a través de la red. Este tipo de actividad si se llevara a cabo en el aula se restringiría únicamente al ámbito académico - docente y compañeros -

Sin embargo, en portugués apenas encontramos este tipo de herramientas, tal y como explicábamos en el apartado de objetivos y metodología, por lo que con este trabajo se pretende darlas a conocer para poder así servir de ayuda a los mediadores.

Por otro lado, sería de gran utilidad que se aprobara una nueva ley de Depósito Legal para documentos electrónicos, regulando y amparando de esta manera la autoría de este tipo de formatos.

Asimismo, no encontramos encuestas específicas que estudien los hábitos de lectura en Portugal de forma periódica ${ }^{26}$, algo que ayudaría también a detectar las áreas de mejora para que las autoridades pudieran incentivarlas en la medida de lo posible. Bien es cierto que en 2017 se lanzó una nueva etapa del Plano Nacional de Leitura, cuyos objetivos para los próximos diez años son fomentar la lectura en diferentes soportes y formar en el acceso a la cultura con recursos propios de las tecnologías de información y comunicación a toda la población: niños, jóvenes y adultos (APEL, 2017). Esperamos pues que en estos años vayan aumentando las propuestas de mediación y formación en este sentido.

No podemos obviar que los más pequeños han nacido con las nuevas tecnologías y tienen una motivación mayor a la hora de usarlas, por lo que pueden servirnos de aliadas para el fomento de la lectura. Pero, no se trata únicamente de poner a disposición de los alumnos nuevos dispositivos de enseñanza, algo que sí que se está haciendo en Portugal desde hace varios años, sino de enseñar a usarlos correctamente y hacerlo con fines educativos. Adecuémonos a las nuevas necesidades de los alumnos acercándonos a ellos a través de unos dispositivos que ellos conocen a la perfección.

En cualquier caso, lo principal es manejarlas de forma adecuada -haciendo uso de la confidencialidad y privacidad en cuanto a protección de datos para menores-, e integrarlas y complementarlas con los documentos en formato tradicional, ya que tan esenciales son unas como otras.

Las nuevas tecnologías deben ser un medio para fomentar la lectura, pero no un fin en sí mismo. Aunque en muchas ocasiones, resulten ser un gran apoyo e incentivo para los niños y jóvenes, no deben ser el objetivo docente.

Cabe destacar que lo primordial de un objeto de lectura es la historia, por lo que todo lo que la acompaña tan solo es relevante en la medida en la que ayude a comprender mejor el contenido o, en su defecto, sirva de medio de animación a la lectura.

\section{BIBLIOGRAFÍA}

AIMC (2018): AIMC niños. Https://www.aimc.es/a1mc-c0nt3nt/uploads/2018/11/ninos2018_informe_principales_ resultados.pdf [consulta: 4 de abril de 2020].

APEL (2017): Plano Nacional de Leitura. Http://www.apel.pt/pageview.aspx?pageid=131\&langid=1\# [consulta: 4 de abril de 2020].

Aprovechar las Tic en la biblioteca infantil (2010): Post Lectura Lab. Http://www.lecturalab.org/print.php?id=2792 [consulta: 4 de mayo de 2020].

Aucadigital (2017): Aucadigital. Barcelona. Www.aucadigital.com [consulta: 4 de mayo de 2020].

BCNmultimedia (2017): Children's Tales. Http://www.childrentales.org [consulta: 4 de mayo de 2020].

BubbleBooks (2017): Tembo el elefante. Http://www.bubblebooks.es [consulta: 4 de mayo de 2020].

Chiquimedia [2017]: Chiquimedia. Http://chiquimedia.org/en/badges\#nurot-release [consulta: 4 de mayo de 2020]

Dadacompany (2017): Marina y la luz. Www.dadacompany.com [consulta: 4 de abril de 2020].

Edokiacademy (2017): Easy stop-motion studio. Http://www.edokiacademy.com [consulta: 4 de junio de 2017].

Fox \& Sheep (2017): Buenas noches HD - La aplicación para niños a la hora de dormir. Http://www.foxandsheep.com [consulta: 4 de abril de 2020].

García Rodríguez, Araceli, y J. A. Cordón García (2013): "De Alicia en el país de las maravillas a las maravillas de Alicia", en Ibersid, 7, 21-32.

26 La última data de 2014 según la Associaçao Portuguesa de Editores e Livreiros (APEL) http://www.apel.pt/pageview.aspx?pageid=112\&langid=1. 
García Rodríguez, Araceli y R. Gómez Díaz (2015): "Las demasiadas aplicaciones: parámetros e indicadores para seleccionar las topapp de lectura para niños", en Anales de Documentación, 18, 2. Http://dx.doi.org/10.6018/ analesdoc.18.2.227071 [consulta: 4 de abril de 2020].

García Rodríguez, Araceli y R. Gómez Díaz (2016): “Niños y apps: aprendiendo a leer y escribir en digital”, en Álabe, 13. Www.revistaalabe.com [consulta: 4 de abril de 2020].

Goodplayguide (2020a): Lucy \& Pogo - Interactive Storybook. Https://www.goodplayguide.com/app/lucy-pogointeractive-storybook [consulta: 4 de abril de 2020].

Goodplayguide (2020b): The Platypus'Search. Https://www.goodplayguide.com/app/the-platypus-search [consulta: 4 de abril de 2020].

Goodplayguide (2020c): Nighty Night Circus. Https://www.goodplayguide.com/app/nighty-night-circus/ [consulta: 4 de abril de 2020].

Goodplayguide (2020d): Dr. Panda Ciudad: Vacaciones. Https://www.goodplayguide.com/app/dr-panda-town-holiday [consulta: 4 de abril de 2020].

Goodplayguide (2020e): Me by Tinybop. Https://www.goodplayguide.com/app/me-by-tinybop [consulta: 4 de abril de 2020]

Goodplayguide (2020f): Tiggly Stamp. Https://www.goodplayguide.com/app/tiggly-stamp [consulta: 4 de abril de 2020].

Goodplayguide (2020g): Princess Drawsalot And The Dragon. Https://www.goodplayguide.com/app/princess-drawsalotand-the-dragon [consulta: 4 de abril de 2020].

Goodplayguide (2020h): Special Stories. Https://www.goodplayguide.com/app/special-stories [consulta: 4 de abril de 2020].

Goodplayguide (2020i): Toca Life: Hospital. Https://www.goodplayguide.com/app/toca-life-hospital [consulta: 4 de abril de 2020].

Goodplayguide (2020j): Easy Studio - Introduction to animation Halloween special. Https://www.goodplayguide.com/ app/easy-studio-introduction-to-animation-halloween-special [consulta: 4 de abril de 2020].

Goodplayguide (2020k): Tiny Builders - Digger, Crane and Dumper for Kids! Https://www.goodplayguide.com/app/tinybuilders [consulta: 4 de abril de 2020].

Goodplayguide (20201): Teach Your Monster to Read. Htps://www.goodplayguide.com/app/teach-your-monster-to-readfirst-steps [consulta: 4 de abril de 2020].

Goodplayguide (2020m): Special Words. Https:/www.goodplayguide.com/app/special-words [consulta: 4 de abril de 2020].

Governo de portugal (1982): Decreto-Lei n. ${ }^{\circ}$ 74/82 - Diário da República n. ${ }^{\circ}$ 51/1982, Série I de 1982-03-03. Https://dre. pt/web/guest/legislacao-consolidada/-/lc/106926278/202005041819/diplomaPagination/1?rp=indice\&q=deposito + le gal+1982\&did=106923228 [consulta: 4 de abril de 2020].

Guillem, José Antonio, J. Celaya (2014): Apps educativas: nuevas formas de acceso al conocimiento, Madrid, Dosdoce.

IFLA (1994): Manifiesto de la IFLA. Http://www.ifla.org/ES/publications/manifiesto-de-la-iflaunesco-sobre-labibliotecap-blica-1994 [consulta: 4 de abril de 2020].

INE (2019): Sociedade da Informação e do Conhecimento - Inquérito à Utilização de Tecnologias da Informação e da Comunicaçãonas Famílias: A proporção de utilizadores de internetbanking aumentou em 10p.p.-2018. Https://www.ine. pt/xportal/xmain?xpid=INE\&xpgid=ine_destaques\&DESTAQUESdest_boui=316295950\&DESTAQUESmodo=2 [consulta: 30 de marzo de 2020].

Informe Tab: estudio sobre el comportamiento de los usuarios de Tablet en España (2013), Logroño, Universidad Internacional de La Rioja. Http://www.tabinnovation.com/informetab [consulta: 4 de abril de 2020].

International Federation of Library Associations, \& P. Gill (2002): Directrices IFLA/UNESCO para el desarrollo del servicio de bibliotecas públicas: Federación Internacional de Asociaciones de Bibliotecarios y Bibliotecas, Organización de las Naciones Unidas para la Educación, la Ciencia y la Cultura. Http://unesdoc.unesco.org/ images/0012/001246/124654s.pdf [consulta: 4 de abril de 2020].

Internet World Stats (2020): European Telecommunications Market Reports. Https://www.internetworldstats.com/europa. htm\#pt [consulta: 30 de marzo de 2020].

The Library of the future (2013): London, Arts Council England. Http://www.artscouncil.org.uk/media/uploads/pdf/The library_of_the_future_May_2013.pdf[consulta: 4 de abril de 2020].

Marques, Pere (2014): "Metainvestigación Dim-Edu 2013-2014 sobre el uso educativo de las tabletas digitales", en Portal de las tabletas digitales y los libros de texto digitales. Http://peremarques.net/tabletasportada.htm [consulta: 4 de abril de 2020].

Mir, Boris (2009): La competencia digital, competencia metodológica. Http://www.xtec.cat/ bmir/competenciasdigitales [consulta: 4 de abril de 2020].

OECD (2019): PISA 2018 Results (Volume I): What Students Know and Can Do, PISA, Paris, OECD Publishing. Https:// doi.org/10.1787/5f07c754-en [consulta: 26 de marzo de 2020].

Payne, Susan, K. Greenberg y M. Walker (2016): The Impact of Computer Usage on Academic Performance: Evidence from a Randomized Trial at the United States Military Academy. Https://seii.mit.edu/wp-content/uploads/2016/05/ SEII-Discussion-Paper-2016.02-Payne-Carter-Greenberg-and-Walker-2.pdf [consulta: 4 de abril de 2020]. 
PORDATA (2020a): Agregados domésticos privados com computador, com ligação à Internet e com ligação à Internet através de banda larga (\%): Qual a percentagem de agregados familiares com internet, com banda larga ou outra conexão mais lenta? Https://www.pordata.pt/Portugal/Agregados+dom\%c3\%a9sticos+privados+com+computador+ + com + liga $\%$ c3 $\% a 7 \%$ c3\%a3o+\%c3\%a0+Internet + e+com+liga $\%$ c3\%a7\%c3\%a3o+\% $\%$ c3\%a0+Internet + atrav $\%$ c3 $\% a 9$ s+de+banda+larga+(percentagem)-1158 [consulta: 26 de marzo de 2020].

PORDATA (2020b): Indivíduos com 16 e mais anos que utilizam computador e Internet em \% do total de indivíduos: por nível de escolaridade mais elevado completo: Qual a percentagem das pessoas com o ensino básico, secundário ou superior a utilizar o computador ou a internet? Https://www.pordata.pt/Portugal/Indiv\%c3\%adduos + com $+16+\mathrm{e}+\mathrm{mai}$ $\mathrm{s}+$ anos + que + utilizam + computador $+\mathrm{e}+$ Internet + em + percentagem + do + total + de + indiv $\% \mathrm{c} 3 \%$ adduos + por $+\mathrm{n} \% \mathrm{c} 3 \% \mathrm{adv}$ el+de+escolaridade+mais+elevado+completo-1141 [consulta: 26 de marzo de 2020].

PORDATA (2020c): Dotações orçamentais públicas para investigação e desenvolvimento (I\&D) em \% do PIB: Quanto prevê, em percentagem do PIB, o Orçamento do Estado para financiamento de I\&D? Https://www.pordata.pt/ Portugal/Dota $\%$ c3\%a7\%c3\%b5es+or\%c3\%a7amentais + p \%c3\%bablicas + para + investiga $\%$ c3\%a $\%$ c 3\%a3o+e+dese nvolvimento+(I+D)+em+percentagem+do+PIB-1099 [consulta: 26 de marzo de 2020].

PORDATA (2019a): Biblioteca Nacional: livros e leitores: Quantos livros e outros documentos tem a BNP? Quantas pessoas ai consultam livros e outros documentos? Https://www.pordata.pt/Portugal/Biblioteca+Nacional + livros $+\mathrm{e}+\mathrm{le}$ itores-806 [consulta: 26 de marzo de 2020].

PORDATA (2019b): Registos de títulos no âmbito do Depósito Legal: Quantas monografias e publicações periódicas são depositadas obrigatoriamente na Biblioteca Nacional de Portugal? Https://www.pordata.pt/Portugal/Registos $+\mathrm{de}+\mathrm{t}$ $\%$ c3\%adtulos+no+\%c3\%a2mbito+do+Dep\%c3\%b3sito+Legal-984 [consulta: 26 de marzo de 2020]

PORDATA (2019c): Computadores com ligação à Internet em \% do total de computadores no ensino básico e secundário: total e por nível de ensino - Continente: Quantos dos computadores nas escolas até ao $4 .^{\circ}, 6^{\circ}, 9^{\circ} .^{\circ}$ ou $12 .^{\circ}$ ano de escolaridade têm internet? Https://www.pordata.pt/Portugal/Computadores $+\mathrm{com}+$ liga $\% \mathrm{c} 3 \% \mathrm{a} 7 \% \mathrm{c} 3 \% \mathrm{a} 3 \mathrm{o}+\% \mathrm{c} 3 \% \mathrm{a} 0+$ Internet + em + percentagem + do + total + de + computadores + no + ensino $+b \% c 3 \% a 1$ sico $+e+s e c u n d \% c 3 \% a 1$ rio + total $+e+p$ or $+\mathrm{n} \% \mathrm{c} 3 \%$ advel+de+ensino+\%e2\%80\%93+Continente-1197 [consulta: 26 de marzo de 2020].

PORDATA (2019d): Títulos em língua portuguesa: total, originais e traduzidos: Quantas monografias originais ou traduzidas para língua portuguesa são catalogadas pela Biblioteca Nacional de Portugal? Https://www.pordata.pt/ Portugal/T\%c3\%adtulos + em $+1 \%$ c3\%adngua+portuguesa+total++originais + e+traduzidos-986 [consulta: 26 de marzo de 2020].

PORDATA (2019e): População residente: total e por grandes grupos etários (\%): Qual a percentagem de jovens, idosos ou pessoas em idade activa? Https://www.pordata.pt/Portugal/Popula $\% \mathrm{c} 3 \% \mathrm{a} 7 \% \mathrm{c} 3 \% \mathrm{a} 3 \mathrm{o}+$ residente + total $+\mathrm{e}+$ por + gra ndes+grupos + et $\%$ c3\%a1rios+(percentagem)-3018 [consulta: 26 de marzo de 2020].

PORDATA (2019f): Rede de Bibliotecas Escolares: número de bibliotecas escolares por subsistema de ensino: Quantas bibliotecas de escolas públicas ou privadas integram o programa rede de bibliotecas escolares? Https://www.pordata. $\mathrm{pt} /$ Portugal/Rede $+\mathrm{de}+$ Bibliotecas + Escolares $+\mathrm{n} \% \mathrm{c} 3 \%$ bamero + de + bibliotecas + escolares + por + subsistema + de + ensi no-1999

Posada Prieto, Fernando (3 de mayo de 2015): Recursos para un proyecto de tabletas. (Blog). Canal TIC: uso educativo de la TIC. Http://canaltic.com/blog/?p=2207 [consulta: 26 de marzo de 2020].

Prioridata (2017): Little Big Genius Books for Kids. Https://www.prioridata.com [consulta: 26 de marzo de 2020].

Scholastic (2016): Kids and Family Reading Report: Finding their Story. Https://www.scholastic.com/content/dam/ KFRR/Downloads/KFRReport_Finding\%20Their\%20Story.pdf [consulta: 26 de marzo de 2020]. 
